\title{
The Use of a Fixation Dressing to Reduce Complications After Neonatal Gastrostomy Tube Placement
}

\author{
Emily H. Steen, ${ }^{1,2}$ Jill M. Tuley, ${ }^{1}$ Swathi Balaji, ${ }^{1}$ Timothy C. Lee, ${ }^{2,3}$ \\ and Sundeep G. Keswani ${ }^{1-3, *}$ \\ 'Laboratory for Regenerative Tissue Repair, Texas Children's Hospital, Houston, Texas. \\ ${ }^{2}$ Department of Surgery, Baylor College of Medicine, Houston, Texas. \\ ${ }^{3}$ Division of Pediatric Surgery, Department of Surgery, Texas Children's Hospital and Baylor College of Medicine, \\ Houston, Texas.
}

Objective: Gastrostomy tubes (GTs) are one of the most common procedures in neonatal surgery, and their malfunction represents one of the most common complaints in the emergency room and clinic. Complications can occur in up to one-third of patients and include pain, peristomal leak, and infection, but can range in severity. We hypothesize that a preventative strategy employing a GT fixation dressing at the time of operation minimizes these postoperative complications in neonates.

Approach: All patients less than 1 year of age who underwent laparoscopic GT placement by a single surgeon in the study period were reviewed. All tubes were secured in place on the external abdominal wall for 2 weeks postoperatively. Demographics and outcomes were evaluated.

Results: Fifty-three percent of our cohort were male, and $47 \%$ were premature. The most common indication for placement was failure to thrive (59\%), and common comorbid conditions were characterized as neurologic (71\%), and cardiac (59\%). The dressing did not prevent hypertrophic granulation tissue formation, but no patient experienced surgical site infection or device-related pressure injury at 30 and 120 days postoperatively. No patient required reoperation or readmission.

Innovation: This simple, one-time, cost-effective fixation dressing has the potential to reduce some of the most common postoperative surgical issues in neonatal patients and can be applied in almost any health care setting.

Conclusions: A dressing aimed at tube fixation and immobilization for the first two postoperative weeks averts some of the major complications of GT placement over a standard follow-up period as compared with the literature.

Keywords: gastrostomy tube, laparoscopic gastrostomy, complications, neonate, dressing

\section{INTRODUCTION}

WITH OVER 14,000 gastrostomy tubes (GTs) placed every year, this operation represents one of the most common pediatric surgical procedures performed in the United States. ${ }^{1}$ Unfortunately, complications of GT placement are common as well:
$11-26 \%$ of patients report pain, peristomal leaks and skin breakdown, tube malfunction, tube dislodgement, tube site infections, and even severe complications like bowel obstruction and peritonitis. ${ }^{2}$ Not only are these complications common, but they are also costly: some estimates approxi-

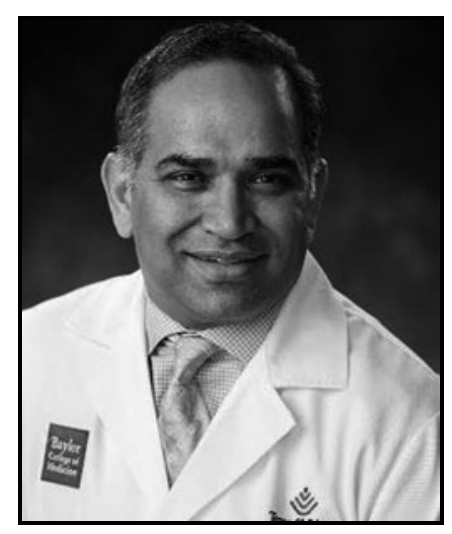

Sundeep G. Keswani, MD, FACS, FAAP

Submitted for publication March 22, 2019. Accepted in revised form April 22, 2019.

*Correspondence: Laboratory for Regenerative Tissue Repair, Texas Children's Hospital, 6701 Fannin Street Suite 1210, Houston, TX 77030 ,

(e-mail: keswani@bcm.edu). 
mate that each return to the clinic or emergency room (ER) costs $\$ 1,200$ per patient. ${ }^{3}$ Not only does this represent a significant annualized financial burden, but it also represents an even more significant quality-of-life issue for caregivers that must repeatedly take their newborn back to the hospital or ER. The pathogenesis of many of these GTassociated complications is thought to be due to excessive movement of the tube in the gastrocutaneous fistulous tract, either due to improper tube fit or to improper tube stabilization, resulting in disrupted and dysfunctional wound repair and subsequent complications.

In this study, we hypothesize that immobilization of a newly placed GT with a simple dressing that minimizes tube movement and avoids devicerelated pressure injuries would ultimately mitigate the long- and short-term postoperative complications typically associated with gastrostomy in neonates, including dislodgement and leakage, while also promoting high-integrity tube site healing. In this study, we define our patient cohort, describe the dressing technique itself, report our outcomes at both 30 and 120 days postoperatively, and then compare those outcomes to the recent literature.

\section{CLINICAL PROBLEM ADDRESSED}

Issues with neonatal GTs are common and represent a cost and quality-of-life burden for caregivers, who are already dealing with the stress of their child's primary medical issues. Immobilization or fixation of the GT in place postoperatively has not yet achieved widespread clinical adoption in children's hospitals. This study is the first to examine the effect(s) of a GT fixation dressing on the incidence of common associated complications at both long and short follow-up intervals. This simple dressing, one that requires no secondary changes after initial placement and one that can be modified and adapted as per the resources available and the clinical setting, has the potential to address a significant clinical issue for these patients and their families.

\section{MATERIALS AND METHODS}

\section{Study design and data acquisition}

This is a retrospective descriptive study of the last 19 consecutive patients to undergo laparoscopic GT placement (CPT-R code 43653) by a single surgeon between September 2015 and December 2018 at Texas Children's Hospital (TCH). These patients then underwent postoperative dressing fixation of the tube for 2 weeks (see placement details below). Other criteria for inclusion were: age under 1 year/neonatal patient sta- tus, and first-time GT placement. Patients were excluded if: GT placement was associated with other major procedures (e.g., fundoplication, gastric tube construct), and incomplete data in the electronic medical record (EMR). Institutional Review Board approval from Baylor College of Medicine was obtained for this study, and the need for written informed consent was waived. Data used for the study were deidentified before analysis.

There are a variety of tube types available and methods by which one can obtain enteral access. To standardize the effect of the tube/button on patient outcomes, the study focused only on the gastrostomy button (MIC-KEY ${ }^{\mathrm{TM}}$; Avanos Medical, Inc.) as this is the most commonly used tube in the neonatal population at our institution for its low profile and ease of use and sizing. Similarly, laparoscopy is the most popular modality by which to place these tubes, for its shorter operating times, smaller incisions, and direct visualization of the stomach and anterior abdominal wall.

Demographic and operative data collected from the EMR for this analysis included: age, gender, race/ethnicity, prematurity (defined as a gestational age at birth $\leq 37$ weeks), weight, primary diagnosis, comorbidities, American Society of Anesthesiologists (ASA) physical status classification, concurrent operative procedures, and GT size. Comorbidities were broadly grouped into neurologic (e.g., seizure disorders, neuromuscular disease, interventricular hemorrhage), cardiac (Tetralogy of Fallot, supravalvular aortic stenosis, double aortic arch, complete atrioventricular canal defect, ventricular septal defect, patent foramen ovale, pulmonary vein stenosis, postheart transplant), pulmonary (bronchopulmonary dysplasia, pulmonary hypertension, meconium aspiration), gastrointestinal (Hirschsprung's, imperforate anus), genitourinary (cloaca, vesicoureteral reflux, postinfectious nephropathy), oropharyngeal (airway teratoma, oral lymphangioma, vascular ring), and congenital or syndromic (Trisomy 21, VACTERL association [vertebral defects, anal atresia, cardiac defects, tracheoesophageal fistula, renal anomalies, and limb abnormalities], Williams syndrome, Denys-Drash, 7q deletion, congenital myotonic dystrophy). Outcome data collected for this analysis included: survival, complications, hospital length of stay (LOS) for the surgical encounter, postoperative LOS, any ER visits pertinent to the GT, any hospital readmissions pertinent to the GT, and any unplanned return to the operating room (OR) pertinent to the GT. Complications included tube dislodgement requiring replacement in pediatric surgery clinic or the ER, pressure injury from the 
feeding tube or bumper, and surgical site infection (SSI), defined as erythema, tenderness, fever, and/ or purulence at the tube site documented in the provider note. Complications also included clinically relevant leaks, defined as evidence of skin irritation (e.g., need for barrier cream, physical exam findings) and in the absence of gastrointestinal motility issues or distal obstruction, and granulation tissue formation. Granulation tissue were considered present if documented in a provider note and if treatment was prescribed or performed for that complication (e.g., triamcinolone cream, silver nitrate cautery, excision). All complications/outcomes were assessed at both 30 (short term) and 120 days (long term) postoperatively; patients could be discharged or still admitted at those time points.

\section{Operative technique}

The laparoscopic GT placement method used was the U-stitch technique described by Georgeson in $1996 .{ }^{4}$ In brief, after peritoneal insufflation, a trocar is placed in the left upper quadrant at the midclavicular line at approximately the intended site for the GT. The greater curvature of the stomach is grasped with a Babcock clamp and brought up to the anterior abdominal wall at the previously selected site and assessed for sufficient distance from the pylorus, as well as for mobility and/or tension. Using 2.0 PDS on CT-1 needle, two transabdominal traction sutures are then placed through the stomach at either side of the clamp; these sutures are then exteriorized and externally secured with hemostats and the trocar is removed. The gastrostomy site is then serially dilated before the button is then placed in the anterior gastric wall through Seldinger technique under direct laparoscopic visualization. The balloon is inflated, and the stay sutures are secured over the button to tack the stomach to the anterior abdominal wall.

\section{Dressing technique}

Immediately following G-button placement, foam dressings (e.g., Mepilex) are positioned circumferentially under the plastic flanges of the port site. This dressing serves to absorb any initial peristomal leaks that might cause skin irritation, helps to prevent pressure injury, and also acts to fix the button in place by disallowing undue twisting of the button in the newly created tract. The feeding tube itself is then connected to the port of the button and foam dressings are then placed under the length of the tube. All tubes are then immobilized flat on the abdominal wall with large transparent adhesive dressings (e.g., Tegaderm) for 2 weeks. The Tegaderm applied in the OR is removed at 5 days such that the stay sutures can be cut, and then is replaced over the Mepilex foam immediately thereafter. Otherwise, the dressings applied in the OR are maintained without disruption over this 2 -week time period. Please see Fig. 1 for a representative image of this dressing.

\section{Statistical analysis}

Categorical statistics are expressed as number (percentage). Continuous statistics are expressed as median (interquartile range $[\mathrm{IQR}]$ ). All data were entered in Microsoft Excel version 15.40 and analyzed using either Excel or the freeware R.

\section{RESULTS}

\section{Demographics and procedure-level factors}

After inclusion and exclusion criteria were applied, a total of 17 patients were found to have undergone laparoscopic GT placement within the 3 years studied. Two patients were excluded for major procedure concurrent to GT placement. Gender was distributed almost equally between male (52.9\%; nine patients) and female (47.1\%; eight patients), and race/ethnicity was majority Hispanic/Latinx (35.3\%) or black/African American (29.4\%), whereas 17.6\% each were either Asian or non-Hispanic white. The median age at the time of surgery was 3.9 months (120 days; IQR: 3.0 months/90 days). The most common indications for GT placement were failure to thrive (58.8\%) and difficulty feeding and/or need for supplemental medications/fluids (23.5\%). Only two patients $(11.7 \%)$ underwent GT placement as an aspiration risk, and one patient (5.9\%) had oropharyngeal obstruction severe enough to require enteric access. Comorbid conditions varied widely, and all

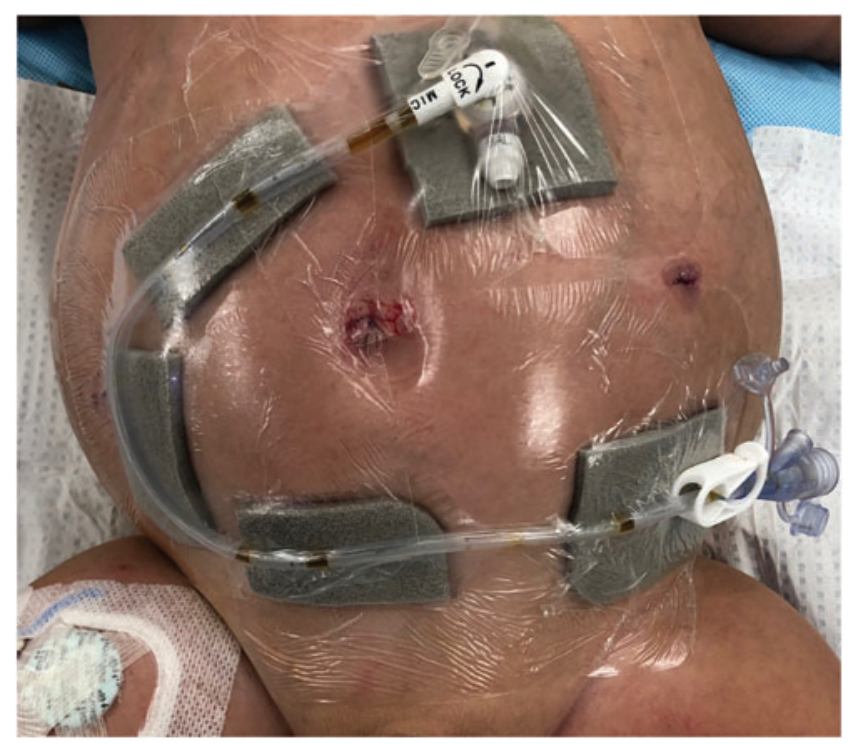

Figure 1. Representative image of fixation dressing. Color images are available online. 
but three patients had more than one. Around 70.6\% of patients had a neurologic or neuromuscular disorder, $58.8 \%$ had cardiac lesions, $52.9 \%$ had a congenital disorder or syndrome, $41.2 \%$ had pulmonary disease, $11.7 \%$ had oropharyngeal abnormalities, and $17.6 \%$ each had gastrointestinal or genitourinary conditions. Many of these comorbidities stemmed from complications of prematurity, which affected $47.0 \%$ of the cohort, which had a median gestational age at birth of 37.5 weeks (IQR: 5 weeks).

The majority ( $82.4 \%$; 14 patients) had an ASA III classification at the time of surgery-the other $17.6 \%$ were classified as ASA IV. The median weight at the time of surgery was $5.3 \mathrm{~kg}$ (IQR: 2.0 ),

Table 1. Characteristics of all patients studied

\begin{tabular}{|c|c|}
\hline & $\mathrm{n}=17$ \\
\hline \multicolumn{2}{|l|}{ Demographics } \\
\hline \multicolumn{2}{|l|}{ Gender } \\
\hline Female & $8(47.1)$ \\
\hline Male & $9(52.9)$ \\
\hline Age (days) at surgery, median (IOR) & $120(90)$ \\
\hline \multicolumn{2}{|l|}{ Race/ethnicity } \\
\hline Hispanic/Latinx & $6(35.3)$ \\
\hline Black/African American & $5(29.4)$ \\
\hline Asian & $3(17.6)$ \\
\hline Non-Hispanic white & $3(17.6)$ \\
\hline \multicolumn{2}{|l|}{ Patient-level factors } \\
\hline \multicolumn{2}{|l|}{ Indications for GT placement } \\
\hline Failure to thrive & $10(58.8)$ \\
\hline Feeding difficulty NOS & $4(23.5)$ \\
\hline Aspiration & $2(11.7)$ \\
\hline Oropharyngeal obstruction & $1(5.9)$ \\
\hline \multicolumn{2}{|l|}{ Comorbidities } \\
\hline Neurologic/neuromuscular & $12(70.6)$ \\
\hline Cardiac & $10(58.8)$ \\
\hline Congenital disorder/syndrome & $9(52.9)$ \\
\hline Pulmonary & $7(41.2)$ \\
\hline Gastrointestinal & $3(17.6)$ \\
\hline Genitourinary & $3(17.6)$ \\
\hline Oropharyngeal & $2(11.7)$ \\
\hline Prematurity $^{a}$ & $8(47.0)$ \\
\hline \multicolumn{2}{|l|}{ Procedure-level characteristics } \\
\hline \multicolumn{2}{|l|}{ ASA class } \\
\hline ASA III & $14(82.4)$ \\
\hline ASA IV & $3(17.6)$ \\
\hline Weight (kg) at surgery, median (IQR) & $5.3(2.0)$ \\
\hline \multicolumn{2}{|l|}{ GT size } \\
\hline 12 French & $3(17.6)$ \\
\hline 14 French & $14(82.4)$ \\
\hline \multicolumn{2}{|l|}{ Concurrent procedures $^{b}$} \\
\hline None & $11(64.7)$ \\
\hline$D L \& B$ & $2(11.7)$ \\
\hline Tracheostomy & $1(5.9)$ \\
\hline Umbilical hernia repair & $1(5.9)$ \\
\hline Other & $4(23.5)$ \\
\hline
\end{tabular}

All data are represented as number (percentage) unless otherwise specified. ${ }^{a}$ Median gestational age at birth of 37.5 weeks (IQR: 5 weeks).

${ }^{b}$ One patient may undergo multiple concurrent procedures

ASA, American Society of Anesthesiologists physical status classification; DL\&B, direct laryngoscopy and bronchoscopy; GT, gastrostomy tube; IOR, interquartile range; NOS, not otherwise specified (need for supplemental medications/fluids). and the tube sizes used were either 12 or 14 French ( $17.6 \%$ and $82.4 \%$, respectively). Minor unrelated procedures performed concomitantly to MIC-KEY button placement included direct laryngoscopy and bronchoscopy, tracheostomy, tympanostomy tube placement, muscle biopsy, umbilical hernia repair, cystoscopy, and pelvic exam under anesthesia. A summary of these results can be seen in Table 1 .

\section{Clinical outcomes}

The median hospital LOS in our patients was 78 days (IQR: 70 days), reflective of the complexity of their medical history and comorbidities. Six patients $(35.2 \%)$ had been admitted since birth, and only one patient (5.9\%) was admitted for GT placement as an outpatient procedure. In general, GT placement tended to occur at the end of an admission, with a median postoperative LOS of 9 days (IQR: 16 days), and all but two patients were discharged at or before 30 days after surgery (88.2\%). No patients experienced complications while admitted, but four patients $(23.5 \%)$ reported issues after discharge within 30 days of surgery: one patient (5.9\%) required an ER visit for GT replacement after dislodgement, whereas three patients (17.68\%) presented to clinic with concerns as to hypertrophic granulation tissue at the tube site. A summary of these outcomes can be seen in Table 2 .

All patients followed up in surgery clinic postoperatively at least twice, although clinic appointments thereafter varied. Ten $(58.8 \%)$ reported problems with the GT within 31-120 days of surgery, resulting in a total of 12 patients $(70.6 \%)$ with complications long term. Not unsurprisingly, the development of any complication within 120 days was related to patient comorbidities: $66.7 \%$ of patients with neurological/neuromuscular issues ( 8 of 12) and syndromic disease (6 of 9) reported an issue(s) after surgery. No patients reported SSI or device-related pressure injuries at 120 days, but two patients had suspected peristomal leak: both were minor, with one patient requiring no treatment and the other prescribed a barrier cream for

Table 2. Overall outcomes

\begin{tabular}{lc}
\hline & $\mathrm{n}=17$ \\
\hline Total hospital LOS (days), median (IOR) & $78(70)$ \\
Postoperative LOS (days), median (IQR) & $9(16)$ \\
Admitted since birth & $6(35.2)$ \\
Outpatient GT surgery & $1(5.9)$ \\
Discharged before 30 days postop & $15(88.2)$ \\
Discharged after 30 days postop $_{\text {Death }^{\text {a }}}$ & $2(11.7)$ \\
\hline
\end{tabular}

\footnotetext{
All data are represented as number (percentage) unless otherwise specified. ${ }^{a}$ Both were attributable to patient comorbidities, not GT placement, and both occurred $>1$ year after discharge.

LOS, length of stay.
} 
skin irritation that resolved upon follow-up. No patients experienced intraperitoneal leak or bleeding. Overall, 70.6\% (12 patients) presented to clinic with complaints of hypertrophic granulation tissue at the site that resulted in management with silver nitrate or triamcinolone. However, only two of these $(11.8 \%)$ were deemed "clinically significant" or "severe" in the EMR (e.g., overgrowth interfered with bumper lay or tube fit), whereas the rest appeared to be treated so as to allay parent concern about appearance. One parent reported tube dislodgement at home that was then expediently replaced in clinic, whereas four patients (23.5\%) returned to the ER for GT replacement after being pulled out, with an overall ER visit rate within 120 days of $29.4 \%$. One patient, however, returned to the ER five times for tube replacement in the year after their surgery (twice within the 120-day time point), reflecting a failure of postoperative teaching resulting in caregiver discomfort with GT management and maintenance. No patients required reoperation for tube malfunction or dislodgment (e.g., unsuccessful replacement, inadvertent intraperitoneal placement) and there were no readmissions pertinent to the GT.

The only subsequent operation performed that related to the GT was for a persistent gastrocutaneous fistula after its removal, occurring greater than 6 months postoperatively. After the primary condition requiring direct enteral feeding resolved, the tube was removed but the tract failed to close by secondary intention. After the primary surgery, two patients $(12.5 \%)$ converted from GT to gastrojejunostomy tubes for persistent reflux, but both conversions occurred 6 months or more after the initial GT placement. Two patients died postoperatively but both events were unrelated to GT placement and occurred over a year postdischarge. Thirty- and 120day outcomes are summarized in Table 3.

\section{DISCUSSION}

Although the purpose for GT placement can be broadly summarized as "inadequate oral intake," the reasons for that diagnosis are quite varied, including failure to thrive, risk of aspiration, oropharyngeal dysfunction or malformation, and any number of congenital and acquired diseases, resulting in a heterogeneous patient population requiring this intervention. With most claiming severe illness or complex comorbidities, however, these infants are among the least equipped to deal with complications that arise from surgery, even those that are considered simple or low impact. For this reason, developing techniques to attenuate postoperative complications in these high-risk neonates takes on a new urgency.
Normal wound healing proceeds as a well-defined series of steps: the initial inflammation phase occurring in the first few days after wounding, then the proliferative phase, where the wound contracts and tissue architecture is reestablished over the course of a few weeks, and finally wound maturation, when collagen remodeling occurs, a process that lasts from months to years. Tube site complications are theorized to start at the midpoint of the wound healing cascade, wherein tube movement causes friction on the skin of the abdominal wall or in the tract itself, leading to a persistent inflammatory reaction that disallows wound healing completion and thus highintegrity tract formation. A vicious cycle then ensues, as improper tube fit from the eroded gastrostomy site further exacerbates tube movement and excessive granulation tissue formation, even leading to peristomal leakage, all of which lead to persistent inflammation and poor healing. Unfortunately, even the most basic activities of daily living, including GT feeding itself, can contribute to excessive tube movement during this critical stage. In this way, the 2 -week duration of the tube site fixation dressing is wholly consistent with what is known about wound healing, as it provides wound support through the majority of the proliferative phase such that a stable dermal tract can form around the tube-a tract that is less susceptible to erosion and other mechanical insults, to the point where most of the common postoperative complications are mitigated.

Complication rates vary widely from study to study in evaluating pediatric GT placement, both because of institutional management styles, surgeon preference, patient population, and, most importantly, in how complications are defined. This becomes particularly apparent with a comparison of our 30- and 120-day outcomes to those of our peer institutions in the clinical literature. These comparisons are valuable in that they theoretically isolate the effect of the fixation dressing itself on GT complication rates, but patient-level differences and significant variability in reporting complications between these studies ultimately limit their utility. In spite of these limitations, however, these studies still provide a context for the results seen in our own cohort.

Bucher's group described a cohort of 381 patients undergoing GT placement using a technique identical to that of our study, but without the postoperative fixation dressing. ${ }^{5}$ Within 30 days of GT placement, they reported an $8 \%$ SSI rate, a $7 \%$ ER visit rate, a $4 \%$ tube dislodgement rate, a $2 \%$ reoperation rate, and 3\% of patients required hospital readmission related to their GT, whereas our rates are $0 \%, 6 \%, 6 \%, 0 \%$, and $0 \%$, respectively. 
However, their studied population was far larger and appeared to have a quite different clinical acuity level, with $46 \%$ of patients discharged within $48 \mathrm{~h}$ of surgery. In addition, they did not report on other common complications, like granulation tissue formation and peristomal leak, and only $56 \%$ of their patients were $<1$ year of age. These comparisons are summarized in Table 4.

Similarly, Sulkowski et al. compared multiple GT procedure types (open Stamm, laparoscopic gastrostomy, percutaneous endoscopic gastrostomy [PEG], laparoscopic PEG, and fluoroscopy-assisted) in terms of common 30-day complication rates with a goal of identifying risk factors for unintentional tube dislodgement. ${ }^{6}$ In this study, laparoscopic GT placement resulted in tube dislodgement in $16 \%$ (vs. $6 \%$ in the current study using a fixation dressing), wound infection in $16 \%$ (vs. 0\%), granulation tissue formation in $36 \%$ (vs. $18 \%$ ), leakage in $28 \%$ (vs. $0 \%$ ), and an ER visit rate related to the GT of $24 \%$ (vs. $6 \%$ ) over a 30-day time point. Despite a more complete accounting of some of the major postoperative complications, only $5 \%$ of their cohort underwent laparoscopic tube placement, and placement technique was not described, nor were the types of tubes used.

In 2014, our own institution sought to describe the financial burden of 30-day GT complications, categorizing concerns either as mechanical (tube dislodgement, tube leak, tube clog, or malfunction) or wound-related (infection, granulation tissue, skin breakdown, bleeding) but without individual anal-

Table 3. Short-term (30-day) and long-term (120-day) complications

\begin{tabular}{lcc}
\hline & $\begin{array}{c}30 \text { Days } \\
(\mathrm{n}=17)\end{array}$ & $\begin{array}{c}120 \text { Days } \\
(\mathrm{n}=17)\end{array}$ \\
\hline Patients with complications overall $_{\text {Granulation tissue }}^{\mathrm{a}}$ & $4(23.5)$ & $12(70.6)$ \\
Tube dislodgement with replacement in the ER $(17.6)$ & $12(70.6)$ \\
Tube dislodgement with replacement in the clinic & $1(5.9)$ & $4(23.5)$ \\
Tube dislodgement requiring replacement in the OR & 0 & $1(5.9)$ \\
Any reoperation for GT malfunction & 0 & 0 \\
SSI/cellulitis & 0 & 0 \\
Device-related pressure injury & 0 & 0 \\
Bleeding & 0 & 0 \\
Tube site leakage & 0 & 0 \\
Intraperitoneal leak & 0 & $2(11.8)$ \\
Number of ER visits & 0 & 0 \\
Readmission rate & $1(5.9)$ & $5(29.4)$ \\
Subsequent reoperation for any reason & 0 & 0 \\
& 0 & $3(17.6)^{\mathrm{b}}$ \\
\hline
\end{tabular}

All data are represented as number (percentage) unless otherwise specified. "Only two patients had granulation tissue deemed "clinically significant" or "severe."

${ }^{\mathrm{b}}$ The only subsequent operation performed that related to the GT was for a persistent gastrocutaneous fistula after tube removal, occurring greater than 6 months postoperatively. Two patients converted from GT to gastrojejunostomy tubes for persistent reflux, but both conversions occurred 6 months or more after the initial GT placement.

$E R$, emergency room; $O R$, operating room; SSI, surgical site infection. ysis of each complication. ${ }^{3}$ This study also included unplanned clinic and ER visits, multiple placement techniques-open, laparoscopic, PEG-and both button (balloon and nonballoon) and standard tube types, again without subdivision of complication rates by clinical setting, surgical technique, or tube type. In this way, an extensive comparison to our current findings could not be performed (Table 5). This analysis, however, did provide a breakdown of costs for an average ER visit related to a gastrostomy concern, reporting an estimated mean cost of $\sim 1,200$ USD per unplanned visit. ${ }^{3}$

Sutherland et al. analyzed 450 pediatric patients with GT, seeking to describe the differences in 120 day complication rates between different surgical techniques. ${ }^{7}$ Open gastrostomy, PEG, laparoscopic gastrostomy with subcutaneous fascial sutures, and laparoscopic gastrostomy with full-thickness transabdominal sutures or T-fasteners ("push technique") were all evaluated, of which only the final group was applicable for comparison with our 120 day data. Complications in the "push group" were not further subdivided into transabdominal suture or T-fastener groups, and they do not distinguish between complications seen within 30 and 31-120 days. Additionally, the median patient age

Table 4. Complications in the literature: 30-day data compared

\begin{tabular}{|c|c|c|}
\hline & $\begin{array}{c}\text { Mason et al. } \\
\mathrm{n}=682\end{array}$ & $\begin{array}{c}\text { Current Study } \\
\mathrm{n}=17\end{array}$ \\
\hline Laparoscopic GT placement & 100 & $17(100)$ \\
\hline Externally fixed suture placement ${ }^{\mathrm{a}}$ & $381(55.8)$ & $17(100)$ \\
\hline Tube dislodgement requiring replacement ${ }^{\mathrm{C}}$ & $14(3.7)^{b}$ & $1(5.9)$ \\
\hline Reoperation & $7(1.8)^{\mathrm{b}}$ & 0 \\
\hline$S S I^{d}$ & $27(7.9)^{b}$ & 0 \\
\hline ER visit rate ${ }^{\mathrm{e}}$ & $27(7.1)^{b}$ & $1(5.9)$ \\
\hline \multirow[t]{2}{*}{ Readmission rate } & $10(2.6)^{b}$ & 0 \\
\hline & $\begin{array}{c}\text { Sulkowski et al } \\
n=468\end{array}$ & $\begin{array}{c}\text { Current Study } \\
\mathrm{n}=17\end{array}$ \\
\hline Laparoscopic GT placement & $25(5.3)$ & $17(100)$ \\
\hline Tube placement technique ${ }^{a}$ & Not reported & \\
\hline Tube dislodgement requiring replacement $\mathrm{f}^{\dagger}$ & $4(16)^{9}$ & $1(5.9)$ \\
\hline Reoperation & 0 & 0 \\
\hline SSI & $4(16)^{9}$ & 0 \\
\hline ER visit rate $\mathrm{e}^{\mathrm{e}}$ & $6(24)^{g}$ & $1(5.9)$ \\
\hline Granulation tissue & $9(36)^{9}$ & $3(17.6)$ \\
\hline Leakage & $7(28)^{g}$ & 0 \\
\hline
\end{tabular}

All data are represented as number (percentage) unless otherwise specified.

${ }^{a}$ The technique descriptors used in this article (externally fixed or temporary suture placement) are alternate terms for the laparoscopic U-stitch approach described in the Methods section.

${ }^{b}$ Percentage of the complication seen in the externally fixed or temporary suture group ( $n=381$ ).

${ }^{\mathrm{c}}$ Representative of replacement in the ER, clinic, or OR.

${ }^{d}$ Sum of SSI $(n=27)$ and stitch abscess $(n=3)$ rates.

${ }^{\mathrm{e} E R}$ visit related to the GT.

fIncludes both severe $(n=3)$ and routine dislodgements.

${ }^{9}$ Percentage of the complication seen in the laparoscopic gastrostomy group $(n=25)$. 
Table 5. Complications in the literature: 30-day data and same institution, compared

\begin{tabular}{lcc}
\hline & $\begin{array}{c}\text { Correa et al. } \\
\mathrm{n}=247\end{array}$ & $\begin{array}{c}\text { Current Study } \\
\mathrm{n}=17\end{array}$ \\
\hline Laparoscopic GT placement & $60(24)$ & $17(100)$ \\
Balloon button placement & $90(36)$ & $17(100)$ \\
ER visit rate & $18(20)^{\mathrm{a}}$ & $1(5.9)$ \\
\hline
\end{tabular}

All data are represented as number (percentage) unless otherwise specified.

${ }^{\text {a }}$ Percentage of complications seen in the balloon button group $(n=90)$.

of the applicable cohort was 13.3 months with an IQR of 61.8-markedly older than our studied group. Overall, they found that the temporary fixation method was associated with less risk of tube dislodgement, decreased rates of granulation tissue formation, and fewer leaks compared with other GT placement strategies. Of the 112 patients undergoing the pertinent technique, $47 \%$ reported development of granulation tissue, $14 \%$ experienced tube site leak, $2 \%$ required reoperation for tube dislodgement, $36 \%$ of patients had an unplanned ER visit, and $15 \%$ were readmitted within 120 days of surgery. This is seen in comparison to our results, which are $71 \%, 12 \%, 0 \%, 29 \%$, and $0 \%$, respectively. SSI was not reported in this study, and tube dislodgement was only reported if it required reoperation, which fails to capture a substantial source of postoperative complications. In addition, only $27 \%$ of patients underwent gastrostomy button placement primarily (vs. standard long GT placement or PEG), and subsequent reported complication rates did not distinguish between these tubes. These comparisons are summarized in Table 6 .

In this study, we found that some of the major postoperative complications of GT placement in neonates were obviated with the use of a fixation dressing for 2 weeks postop, including SSI, peristomal leaks, the need for reoperation, and the need for readmission for tube malfunction or wound concern. In addition, our ER visit rates at both 30 and

Table 6. Complications in the literature: 120-day data compared

\begin{tabular}{lcc}
\hline & $\begin{array}{c}\text { Sutherland et al. } \\
\mathrm{n}=450\end{array}$ & $\begin{array}{c}\text { Current Study } \\
\mathrm{n}=17\end{array}$ \\
\hline Laparoscopic transabdominal suture technique & $112(24.9)^{\mathrm{a}}$ & $17(100)$ \\
G-button/low-profile tube placement & $121(26.9)$ & $17(100)$ \\
Tube dislodgement requiring reoperation & $2(1.8)^{\mathrm{b}}$ & 0 \\
ER visit rate & $38(36)^{\mathrm{b}}$ & $5(29.4)$ \\
Readmission rate & $5(15)^{\mathrm{b}}$ & 0 \\
Granulation tissue & $53(47.3)^{\mathrm{b}}$ & $12(70.6)$ \\
Leakage & $16(14.3)^{\mathrm{b}}$ & $2(11.8)$ \\
\hline
\end{tabular}

\footnotetext{
"The technique descriptor used in this article (the "push" technique) is an alternate term for the laparoscopic U-stitch approach described in the Methods section, comprising the use of both external transabdominal sutures (80 patient) and T fasteners (32 patients).

bPercentage of the complications seen within the "push" technique group (112 patients).
}

120 days were lower than those of our counterparts, most strikingly in comparison to those of our own institution just 5 years ago. However, in contrast, nonoperative tube dislodgement was a very common complication for our cohort, occurring in $29 \%$ of our patients at 120 days, resulting in five unplanned ER visits-none of which resulted in the need for reoperation. This may not, however, be appropriately matched to the literature: the reportage rates for this complication in other studies were unusually low, often only noted if the dislodgement required operative intervention. We also found the rate of identification and intervention for hypertrophic granulation tissue in our cohort to be quite high in comparison to other groups in the literature. This appears to reflect how aggressive our institution is about treating granulation tissue when the caregiver is apprehensive, even if not truly clinically significant-only two patients had actual mechanical or wound healing issues secondary to this tissue overgrowth, and none ultimately required invasive management, like excision or tube resiting.

The major limitations to this study include its retrospective nature, small sample size, and single surgeon experience, and thus is subject to the biases associated with this type of data collection. In addition, we depended on documentation of a clinical diagnosis of these complications by a medical provider, which often was not a surgeon (e.g., neonatologist or pediatrician while the patient was admitted, physician assistants in surgery clinics), and treatment practices may not align with surgeon experience and management preferences. Patients may also present to other hospitals for their postoperative care, and thus these results may underestimate ER visit rates. Despite these limitations, we feel that our outcomes are thought provoking enough, and the issue critical enough, to expand the focus of this study into a prospective observational trial of GT dressing protocolization at our institution, as well as identifying the optimal duration of tube fixation, so as to inform future best postoperative practices. Furthermore, while a small study, we reported all common complications, no matter how minor they are often perceived to be-something that provides a uniquely granular view of the day-to-day challenges of GT management.

In summary, we demonstrate that a secure fixation dressing maintained for 2 weeks represents a practical preventative strategy for some of the major postoperative complications of GT placement over two standard follow-up periods. In addition, this strategy reduces wound care costs and patient/caregiver anxiety regarding dressing changes in the immediate postoperative period. There are many 
burdens-both financial and temporalinherent to the care of the neonatal GT patient, and thus any modality, even if not ideal, by which one can enhance postoperative GT outcomes and ensure durable long-term direct enteric access would prove a marked advancement in the quality of life of both children and their caregivers.

\section{INNOVATION}

GT placement is a common pediatric surgical procedure in the neonatal population, but postoperative complications are frequent. In this article we show that a simple, inexpensive dressing that securely affixes the tube to the abdominal wall of the patient and is maintained in place for the 2 weeks following surgery is associated with reduced complication rates as compared with those seen in the recent literature. It can be easily incorporated into postoperative bundles and markedly simplifies wound care and costs, while decreasing the financial and emotional burden to the patients and their families.

\section{ACKNOWLEDGMENTS AND FUNDING SOURCES}

None declared.

\section{AUTHOR CONTRIBUTIONS}

E.H.S. and J.M.T. collected the data; E.H.S. analyzed the data; E.H.S. drafted the article; E.H.S., J.M.T., S.B., T.C.L., and S.G.K. provided critical edits to the article, S.G.K. designed the study.

\section{AUTHOR DISCLOSURE AND GHOSTWRITING}

No competing financial interests exist. The authors reported no proprietary or commercial in- terest in any product mentioned or concept discussed in this article. The content of this article was expressly written by the author(s) listed. No ghostwriters were used to write this article.

\section{ABOUT THE AUTHORS}

Emily H. Steen, MD, is a general surgery resident at Baylor College of Medicine completing her research fellowship in the Laboratory for Regenerative Tissue Repair at Texas Children's Hospital. Jill M. Tuley, MS, is the laboratory manager of the Laboratory for Regenerative Tissue Repair at Texas Children's Hospital. Swathi Balaji, PhD, is the co-principal investigator of the Laboratory for Regenerative Tissue Repair at Texas Children's Hospital and assistant professor in the Division of Pediatric Surgery at Baylor College of Medicine. Timothy C. Lee, MD, is a fetal surgeon and director of the Colorectal and Pelvic Health Program at Texas Children's Hospital. Sundeep G. Keswani, MD, is the principal investigator of the Laboratory for Regenerative Tissue Repair, the Surgical Director of Basic Science Research and Professor of Surgery, Pediatrics, and Obstetrics and Gynecology at Baylor College of Medicine and Texas Children's Hospital.

\section{REFERENCES}

1. Baker L, Emil S, Baird R. A comparison of techniques for laparoscopic gastrostomy placement in children. J Surg Res 2013;184:392-396.

2. Pemberton J, Frankfurter C, Bailey K, Jones L, Walton JM. Gastrostomy matters - the impact of pediatric surgery on caregiver quality of life. $J$ Pediatr Surg 2013;48:963-970

3. Correa JA, Fallon SC, Murphy KM, et al. Resource utilization after gastrostomy tube placement: defining areas of improvement for future quality improvement projects. J Pediatr Surg 2014;49:1598-1601.

4. Sampson LK, Georgeson KE, Winters DC. Laparoscopic gastrostomy as an adjunctive procedure to laparoscopic fundoplication in children. Surg Endosc 1996;10:1106-1110.

5. Mason CA, Skarda DE, Bucher BT. Outcomes after laparoscopic gastrostomy suture techniques in children. J Surg Res 2018;232:26-32.

6. Sulkowski JP, De Roo AC, Nielsen J, et al. A comparison of pediatric gastrostomy tube placement techniques. Pediatr Surg Int 2016;32: 269-275.

7. Sutherland C, Carr B, Biddle KZ, Jarboe M, Gadepalli SK. Pediatric gastrostomy tubes and techniques: making safer and cleaner choices. J Surg Res 2017;220:88-93.
Abbreviations and Acronyms

$$
\begin{aligned}
\mathrm{ASA} & =\text { American Society } \\
& \quad \text { of Anesthesiologists } \\
\mathrm{EMR} & =\text { electronic medical record } \\
\mathrm{ER} & =\text { emergency room } \\
\mathrm{GT} & =\text { gastrostomy tube } \\
\mathrm{IOR} & =\text { interquartile range } \\
\mathrm{LOS} & =\text { length of stay } \\
\mathrm{OR} & =\text { operating room } \\
\mathrm{PEG} & =\text { percutaneous endoscopic } \\
\mathrm{SSI} & =\text { gastrostomy } \\
& \text { surgical site infection }
\end{aligned}
$$

